\title{
Classification and Segmentation of fMRI Spatio- Temporal Brain Data with a NeuCube Evolving Spiking Neural Network Model
}

\author{
Maryam Gholami Doborjeh, Elisa Capecci and Nikola Kasabov \\ Knowledge Engineering \& Discovery Research Institute (KEDRI) \\ Auckland University of Technology \\ Auckland, New Zealand \\ mgholami@aut.ac.nz
}

\begin{abstract}
The proposed feasibility analysis introduces a new methodology for modelling and understanding functional Magnetic Resonance Image (fMRI) data recorded during human cognitive activity. This constitutes a type of Spatio-Temporal Brain Data (STBD) measured according to neurons spatial location inside the brain and their signals oscillating over the mental activity period [1]; thus, it is challenging to analyse and model dynamically. This paper addresses the problem by means of a novel Spiking Neural Networks (SNN) architecture, called NeuCube [2]. After the NeuCube is trained with the fMRI samples, the 'hidden' spatio- temporal relationship between data is learnt. Different cognitive states of the brain are activated while a subject is reading different sentences in terms of their polarity (affirmative and negative sentences). These are visualised via the SNN cube (SNNc) and then recognized through its classifier. The excellent classification accuracy of $90 \%$ proves the NeuCube potential in capturing the fMRI data information and classifying it correctly. The significant improvement in accuracy is demonstrated as compared with some already published results [3] on the same data sets and traditional machine learning methods. Future works is based on the proposed NeuCube model are also discussed in this paper.
\end{abstract}

Keywords- fMRI; NeuCube; Spatio-Temporal Brain Data; Evolvings Spiking Neural Networks;

The human brain processes input information across different evoked cognitive states, acting as a complex information processing machine [1]; therefore, a proper model is needed that is capable of tracing this information and the mental processes that generate it. Over the past decades, a variety of imaging techniques have been developed to address this challenge and analyse the spatio-spectro temporal activity. They differ in what they can measure and what level of spatial and temporal resolution they can obtain [4]. Some of these techniques are Magneto Encephalography (MEG), Electro Encephalography (EEG) and fMRI. MEG and EEG are based on magnetic and electrical activity of the brain and possess high temporal resolution but unclear localization (i.e. brain signals are recorded in the sequence of milliseconds) [4]. In contrast, fMRI data is defined as a powerful STBD that presents neuron localization with high resolution [4]. This data is based on magnetic resonance, which can be affected by blood flow changing against neuronal activities. In fact, active neurons demand high level of oxygen to start firing, which is carried by blood cells. By increasing the number of spikes in activated brain regions, blood flow will be increased in those particular parts; therefore, fMRI data is based on Blood Oxygenation Level Dependent (BOLD) contrast [5]. Patterns of voxels activity are influenced by BOLD responses, while different regions of the brain are activated by means of different stimulus. The fMRI data provides the spatial localization of the brain in a three dimensional cell (i.e. a voxel) and the temporal information as the fourth dimension. Each of these voxels represents fluctuation of BOLD intensity of thousands of neurons over time. Additionally, the fMRI technique is not considered harmful on subjects. All of these reasons, brought researchers to choose fMRI technique to study the brain functional activities. There are numerous common objectives pursued in fMRI data analysis, including: (1) localizing the activated brain regions during a particular mental task; (2) detecting the brain information pathways corresponding to the functional activities; and (3) predicting disease or psychological states [4].

In this paper, available online fMRI data [6] of one subject is analysed to study how affirmative/negative sentences affect the neural activities and whether different sentences in term of their polarity can be recognized or not.

To achieve these goals, we have used the recent proposed NeuCube SNN architecture [1] to perform multi voxel patterns analysis of the fMRI data. The spiking neurons of the cube are able to evolve new connections according to the temporal information 'hidden' in data. This information can be visualised in the SNNc and the output classified. An evolving SNN (eSNN) is also used in output classifier [7, 2].

In the following section, the NeuCube-based model is presented. Then, in section II the model design and implementation is described. In section III. A, dynamic brain activities are visualised. In section III.B the classification outputs are presented and compared with already published results on the same data set and also traditional Machin learning methods. Finally, conclusion and future works are presented in section IV and V respectively.

\section{A SPIKING NEURAL NETWORK METHODOLOGY FOR MODELLING FMRI SPATIO-TEMPORAL DATA OF BRAIN COGNITIVE ACTIVITIES}

\section{A. Spiking neural network for modelling STBD}

The brain processes the input information in the form of temporal binary events called spikes [1]. 
Spiking Neural Networks (SNNs) are inspired from a biologically realistic model of the brain that processes the dynamic input information across a large number of spiking neurons. SNNs are considered as the third generation of neural networks. In this network, during the learning process, the connection weights between neurons are modified by transferring the spikes across the synaptic connections. SNNs structure have been used in many approaches, such as encoding the continuous data into trains of spikes [8] and data learning [9, $10,11]$. Some of their remarkable features are: compact representation of space and time; fast data learning; time-based and frequency-based information representation [2, 12]. Due to these reasons, SNNs can be considered as one of the best techniques for STBD analysis, such as fMRI data.

In this study, we used a NeuCube-based model for cognitive fMRI data learning, classification and understanding. This model used an eSNN approach during its learning process and a dynamic evolving SNN approach (deSNN) in its classifier. These two methods are briefly introduced in the following.

1) The eSNN paradigm extends the evolving connectionist system models with the use of integrative and fire model (IFM) of a neuron and Rank-order (RO) learning [2]. The RO learning motivation is based on the assumption that most important information of an input pattern is contained in earlier arriving spikes [13]. It establishes a priority of inputs (synapses) based on the order of the spike arrival on these synapses for a particular pattern, which is a phenomenon observed in biological systems [2, 13, 14].

2) deSNN: based on this learning rule, for every training sample, a new output neuron is created and connected to the all neurons of the cube. Their connection weights are initially set to zero and then established by RO rule. Also they will be dynamically modified by further incoming spike trains.

\section{B. Brain-like NeuCube for fMRI data modelling}

For fMRI data implementation, we used NeuCube as a computational model, presented in a software simulator written in MATLAB. The NeuCube is a brain-inspired evolving spiking neural network architecture for STBD analysis and knowledge extraction from the data and the brain processes that generates it [15]. A representation of the NeuCube for fMRI data modelling and classification is shown in Fig.1.

This NeuCube consists of three significant modules: fMRI data encoding and mapping; unsupervised learning and brainlike SNNc visualisation; supervised learning and classification output validation.

- In the first module, data is spatially mapped to a 3D cube, according to the voxel coordinates. Then, preselected voxel activity patterns of particular brain regions are encoded into spike trains using Address Event Representation (AER) algorithm [14, 16] and later fed into the SNNc via inputs neurons.

- $\quad$ In the evolving SNNc module, unsupervised learning is performed using Spike-Timing Dependent Plasticity (STDP) learning rule [2]. In this module, the spatiotemporal relationship between data are encoded to the connection weights. These weights are influenced by the temporal relationship between two active neighbour neurons. In fact, when the spike trains are continuously fed into the $\mathrm{SNNc}$ in their temporal order, the connection weight between two neurons will increases or decreases depending on the timing of post-synaptic action potential in relation to the pre-synaptic spike. Consequently, all connection weights are established to the trained network.

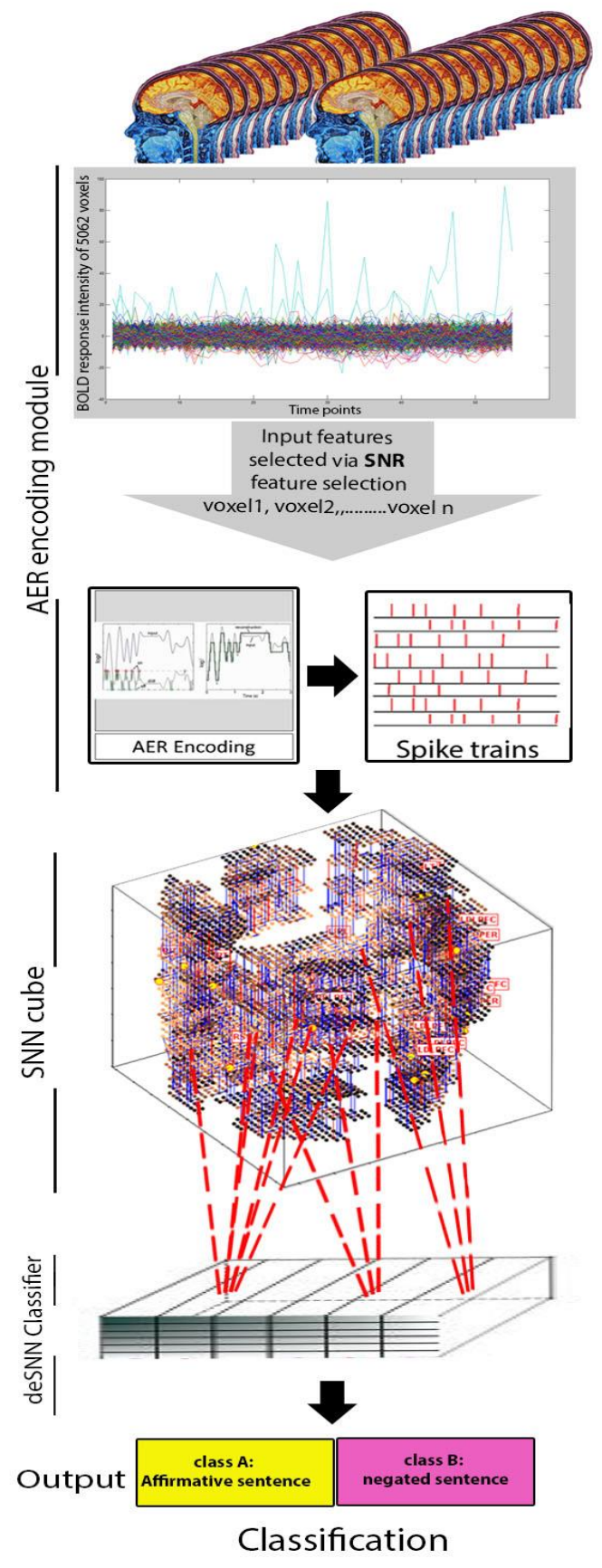

Fig. 1. A block diagram of the NeuCube architecture with its main modules for a case study on fMRI

- In the output classification module, supervised learning of the spike sequences is performed using deSNN algorithm [17]. When a new data without class label 
(test data) is propagated to the network, an output neuron is created and its connection weights are calculated. These new connection weights will be compared with those ones generated during the unsupervised learning. Then testing data will be labelled with the label of existing neurons that have closest connection weights. Therefore, the same neurons are spiking to recall the same temporal connectivity that was created during the unsupervised learning [18].

In this study, the output neurons learnt to classify the voxel activity patterns of the fMRI data into two cognitive states (class 1: the brain activity patterns while the subject is reading affirmative sentences and class 2 : the brain activity patterns while the subject is reading negated sentences).

\section{MODEL DESIGN AND IMPLEMENTATION}

The principle represented in Fig. 1 is applied to visualise and classify the cognitive fMRI data through the following procedure:

1. Task-related fMRI cognitive data is selected amongst the online available [6].

2. Particular brain regions of interest, which are activated during the mental task are detected.

3. Selected voxels of the fMRI data are encoded into trains of spikes.

4. The whole brain voxels are mapped into the SNNc and input neurons are allocated according to the input feature coordinates.

5. Unsupervised training of the $\mathrm{SNNc}$ is performed [17].

6. The spiking activity and the neuron connections are visualised according to the voxel activity patterns generated by affirmative/negated sentences.

7. Supervised training of the classifier is performed and classification results are then evaluated.

8. The classification accuracy is optimized using grid search method and the best accuracy is reported.

A flowchart of the fMRI data analysis via NeuCube is illustrated in Fig. 2.

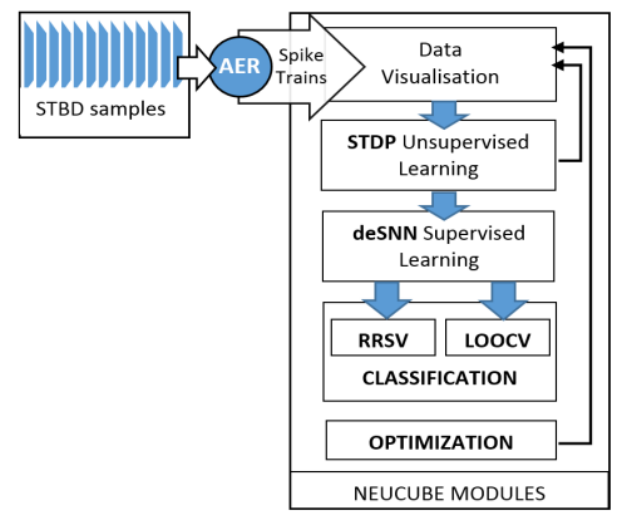

Fig. 2. A flowchart of the NeuCube framework. STDP learning rule is used during the NeuCube training and deSNN method is used in classifier.

\section{A. fMRI data description}

The known Star/ Plus fMRI data set, originally collected by Marcel Just and his colleagues in Carnegie Mellon University's CCBI [6, 19], was selected for this experimental study. Star/plus fMRI data sets are sequences of images from the whole brain volume captured every 500 milliseconds, while human subjects were undertaking a cognitive task. This task-related fMRI data set consists of a collection of trials. Each trial is started by presenting a stimuli (picture or sentence) that remains on the screen for 4 seconds. Then, a blank screen appears for another 4 seconds. After that, second stimuli (picture or sentence) is presented for the next 4 seconds. Subjects are supposed to press the button 'Yes' or 'No' whether a sentence describes the picture correctly or not. Finally, each trial is followed by 15 seconds of resting period before the beginning of the next trial. Each trial takes approximately 27 seconds corresponding to $54 \mathrm{fMRI}$ data points (two brain images per second). The brain is partitioned into 27 distinct regions of interest (ROIs), each corresponding to different voxel activity patterns.

When the subject is reading different sentences on the screen, different areas of the brain are activated with respect to the sentence polarity. Therefore, in this study, we selected the most informative voxels associated with brain regions activated by reading trials to run the experiments with the NeuCube-based model.

\section{B. Affirmative vs negative sentence trials}

In order to analyse the brain activity generated by sentence stimuli, the more corresponding activated voxels were extracted from the whole fMRI data. We selected the brain images captured during 40 trials. The first 20 trials started with a picture stimuli, which remains on the screen for 4 seconds, equal to 8 fMRI sequences ( 2 images per seconds). That was followed by 4 seconds rest, which is equal to another 8 fMRI sequences. Therefore, the onset of the sentence presentation is the $8^{\text {th }}$ second of the trial or the $16^{\text {th }}$ brain image (images from $i=17$ to $i=32$ ). The other 20 trials started with a sentence stimuli which remains on the screen for 4 seconds. That was followed by 4 seconds rest. Therefore, the onset of the sentence presentation is the $0^{\text {th }}$ second of the trial or the $1^{\text {th }}$ brain image $(i=1$ to $i=16)$.

\section{An fMRI features selection approach}

An fMRI case study contains a huge number of voxels, according to the different brain data sizes. To analyse the voxel activity patters of the activated ROIs, the most suitable features need to be chosen. To create our computational model, we have selected the most appropriate features using Signal-to-Noise Ratio (SNR) feature selection [7, 20]. SNR evaluates how appropriate a variable is to distinguish samples fitting to dissimilar classes [7]. Features are ranked with respect to their SNR values. The SNR results were obtained using the available online NeuCom platform [21]. The NeuCom is a generic knowledge engineering environment for data analysis, modelling and knowledge discovery developed by the Knowledge Engineering and Discovery Research Institute KEDRI [21]. 


\section{D. fMRI data mapping to the SNNc of NeuCube}

In order to prove the scalability of NeuCube to adapt the SNNc for different brain structures and data size, we visualised the fMRI data using two different brain mappings (M1: star/plus fMRI coordinates and M2: Talairach- based coordinates [22]).

M1: The whole fMRI voxels were spatially mapped into the SNNc, which can evolve according to the different number of voxels (data size). Then, every pre-selected voxel allocated an input neuron to transfer its activity patterns.

M2: Star/Plus coordinates can also be converted into Talairach- based coordinates [22]. We transformed the coordinates of the pre-selected voxels and mapped them into a NeuCube of 1471 spiking neurons. Each of these neurons represents the centre coordinate of a one cubic centimetre area from the 3D Talairach Atlas. To map the star/plus fMRI coordinates of the informative voxels, we detected the activated brain regions containing these voxels. Each brain region is defined as a Brodmann area with distinct Talairach coordinate inside the brain. For every voxel, we found the nearest Talairach-based coordinate in the relevant Brodmann area and mapped it to the cube. The 3D size of the cube is able to change to adapt the data dimension. We obtained a cube of $51 \times 56 \times 8$ spiking neurons corresponding to the maximum values of $x, y$ and $z$ coordinates from the Star/Plus fMRI data. Fig. 3 illustrates the mapping of the fMRI data into the SNNc (according to the Star/Plus and Talairach-based coordinates). The 20 input neurons were allocated and labelled with their corresponding brain region's names.

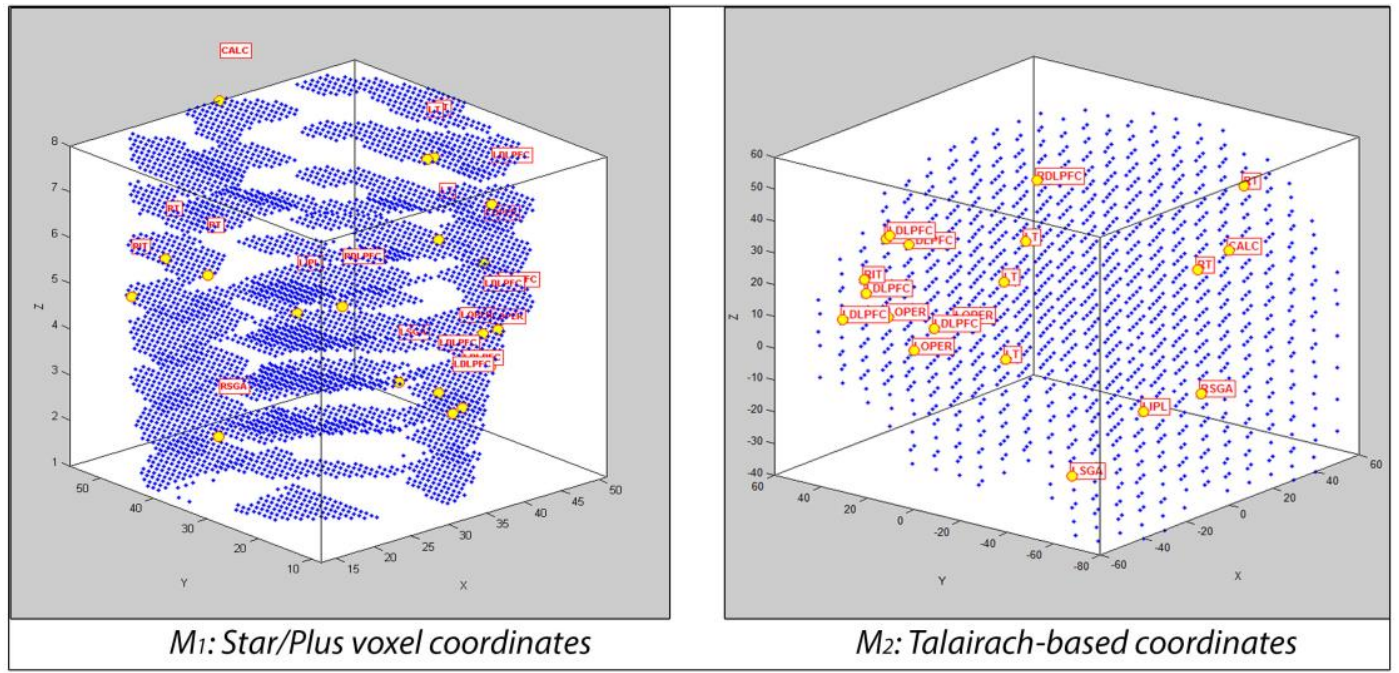

Fig. 3. $\mathrm{M}_{1:}$ the whole fMRI data loaded into the SNNc based on the star/ plus coordinates and then 20 pre-selected voxels were allocated and labelled by brain region names; $\mathrm{M}_{2:}$ the 20 pre-selected voxels were mapped into the 1471 spiking neurons of the cube with respect to their Talairach coordinates.

To transfer the STBD to the SNNc, AER encoding model was applied on the input voxel activity patterns. In this approach, if a voxel BOLD intensity value exceeds the AER threshold, a spike occurs [14]. Fig.4 represents an example of 5 voxel activity patterns were transferred into the 5 spike trains during 16 fMRI time series. The spike trains represent the time relationship between neurons of the SNNc, which is

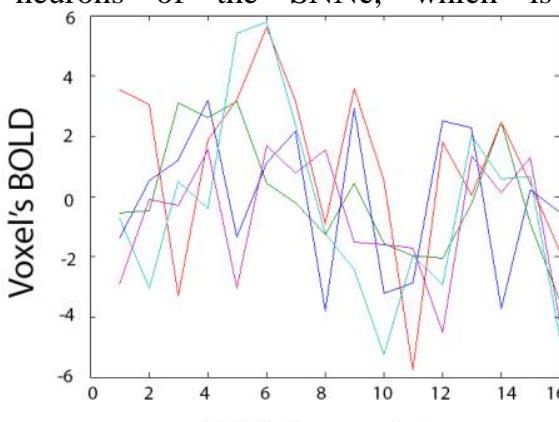

implemented using leaky integrate and fire model of the neurons. After the fMRI data was fed into the SNNc, neurons were trained using STDP learning rule [2] during an unsupervised learning. Therefore, neurons learnt from spatiotemporal patterns of the input fMRI data to create new neuron connections.
fMRI time points

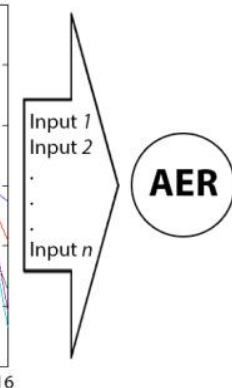

Fig. 4. An example of 5 voxel activity patterns encoded to spike trains via AER encoding algorithm.

\section{E. The NeuCube parameter setting}

NeuCube is a stochastic method, which means that the initial connections between the neurons of the cube are randomly created. Also, the model is highly influenced by parameter setting. Some of the most important parameters of the model are explained in the following. 
- $\quad$ AER encoding threshold used to transfer the input fMRI voxel activity patterns into the spike trains.

- The connection distance between neurons of the SNNcOnce the spike trains of the fMRI data were fed into the NeuCube, the initial connection between the input neurons and their neighbours were created based on this distance parameter (i.e. The initial connections of the SNNc are based on the small world connection rule where: each neuron in the cube is connected to its nearby neurons which are within a distance $d$, where $d$ is equal to the longest distance between any pair of neurons in the cube [23]).

- The STDP rate parameter [17]- According to this learning rule, the connection weight is influenced by the temporal relationship between two active neighbour neurons. In fact, their connection weight will increases or decreases depends on the order of neuron firing.

- The deSNN classifier parameters- (mod and drift parameters). As explained in [2], an output neuron is evolved for every fMRI training sample and connected to all neurons in the SNNc. The initial connection weights of the output neurons are set to zero. The weight of every new connection is based on RO learning rule. This is calculated depending on a modulation factor ( $m o d)$ of the order of the incoming spikes. The new connection weights will then increase or decrease according to the number of spikes that follow the first one (drift). We have set these parameters values of 0.4 and 0.25 respectively.

\section{EXPERIMENTAL RESULTS}

As mentioned in section II.C, we have used SNR tool (available through NeuCom) to select the most important voxels containing the BOLD responses generated by sentence stimuli. Fig. 5 illustrates the fMRI voxels ranked by their SNR values. The voxel with higher SNR value represents the feature with most informative activity patterns.

In this study, we have used 20 of these voxels to allocated 20 input neurons inside the SNNc for the STBD transferring.

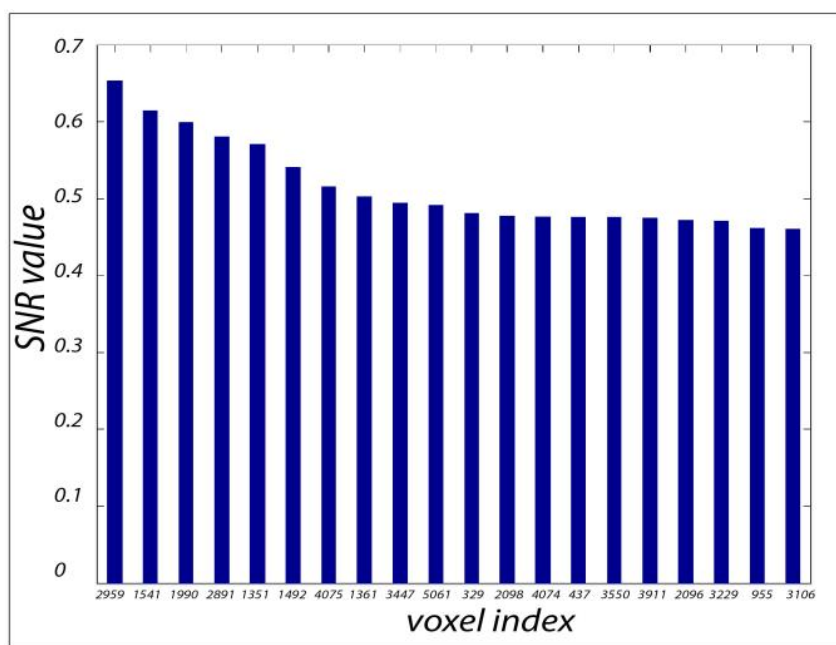

Fig. 5. The first 20 important voxels based on SNR results (voxel number 2959 is ranked as the highest.
Table 1 reports the 20 pre-selected voxels and their corresponding ROIs according to the voxels geometric coordinates.

Table I. More activated ROIs are presented in decreasing order of SNR values. In bracket is the number of voxels located in every region

\begin{tabular}{|l|l|c|l|l|l|}
\hline \multicolumn{5}{|c|}{ Activated brain region (number of selected voxels) } \\
\hline $\mathbf{1}$ & 'LT' (3) & $\mathbf{4}$ & 'LDLPFC' (6) & $\mathbf{7}$ & 'RDLPFC'(1) \\
\hline $\mathbf{2}$ & 'LOPER' (3) & $\mathbf{5}$ & 'RT'(2) & $\mathbf{8}$ & 'RSGA' (1) \\
\hline $\mathbf{3}$ & 'LIPL'(1) & $\mathbf{6}$ & 'LSGA'(1) & $\mathbf{9}$ & 'RIT'(1) \\
\hline
\end{tabular}

We can conclude from the Table 1 that when a subject is making a decision about sentence polarity, more numbers of activated voxels (6 of them) are located on the Left Dorsolateral Prefrontal Cortex (LDLPFC). However, there are 8 more brain regions associated with this mental activity. In fact, if we look at the SNR results, the Left Temporal (LT) region obtained the highest SNR value followed by the LOPER region and then the Inferior Parietal Lobule (LIPL) region.

We have taken into account this information to designed three different experiments (or sessions), as explained in the following:

- Session I: 20 voxels were selected from a combination of 9 active brain ROIs, as reported in Table 1.

- $\quad$ Session II: 20 voxels were selected only from the Right Dorsolateral Prefrontal Cortex (RDLPFC) which was one of the most activated brain regions during the sentence reading trials (resulted from "LDLPFC(6)" written in Table 1). In this experiment, we used only these voxels as inputs to the SNR algorithm to select the most informative ones. Then, 20 of them were selected.

- Session III: 20 voxels were selected from the LDLPFC region via SNR feature selection.

\section{A. The NeuCube visualisation for spatio-temporal connections based on the fMRI spiking activity}

After the NeuCube learning process, the spatio-temporal relationship between different brain regions was analysed with respect to the data collected. For this study, the fMRI data was divided into two classes according to the sentence polarity (class 1 - the subject is reading an affirmative sentence; class 2 - the subject is reading a negative sentence). The evolvability of the model is realised in 2 ways: new input variables and data can be used for the SNNc training; new class labels can be evolved in the output classifier eSNN. Fig. 6 and Fig. 7 represent the 1471 neurons of the brain-like cube were mapped according to the Talairach brain atlas. The dynamic brain activities were captured inside the SNNc, while the spiking neurons were trained with two fMRI time series (affirmative versus negative sentences) respectively. Blue lines are positive spikes while the red lines are negative spikes. The brighter the colour of a neuron, the stronger its activity with a neighbour neuron. Thickness of the lines also identify the neuron's enhanced connectivity. We can conclude from these figures that the subjects performed differently for different complex mental tasks. The more and stronger connections were formed between the neurons located in the left hemisphere (LDLPFC and LT) than in the right hemisphere (RDLPFC and RT). The connectivity was especially 
enhanced between the input neurons (i.e. the selected voxels) of the LDLPFC and LOPER regions. Also, the spatio-temporal relationship between these neurons can be segmented as a homogeneous region in terms of the spatial correlation between neighbouring neurons and their temporal spiking activities over the time. Fig. 8 illustrates a segmentation of the activated brain regions captured via the NeuCube network analysis.

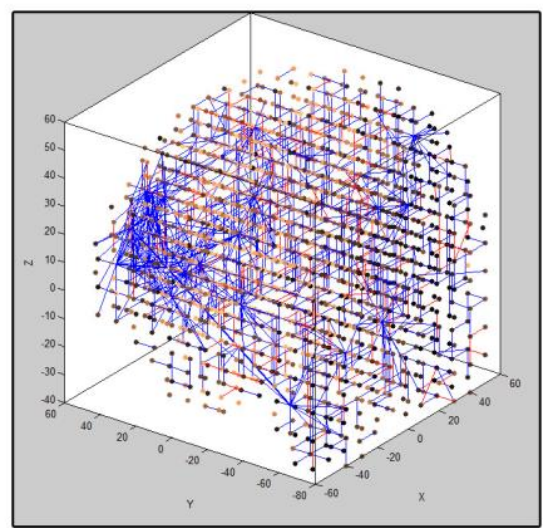

Fig. 6. Neuron connections generated by affirmative sentences.

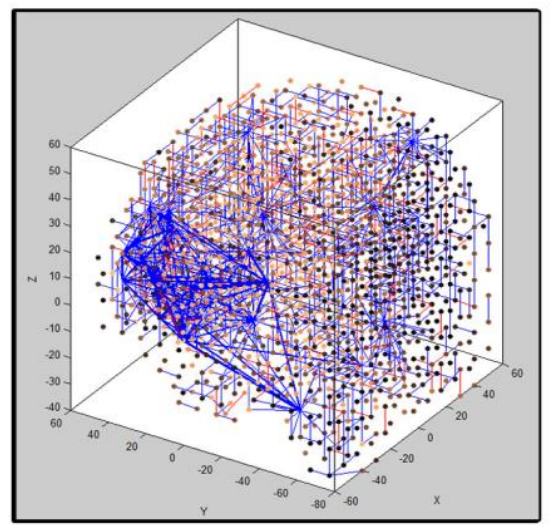

Fig. 7. Neuron connections generated by negative sentences.

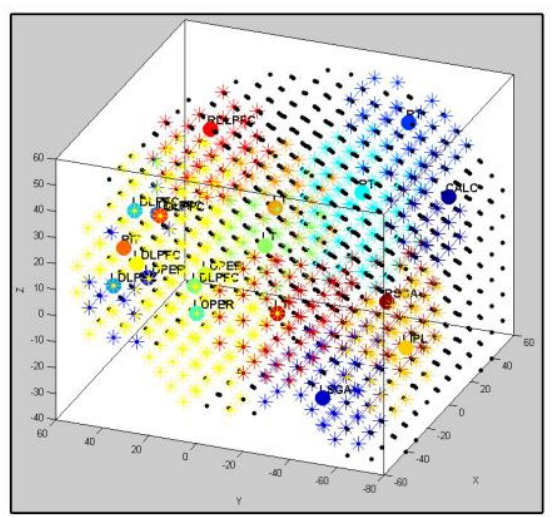

Fig. 8. The NeuCube SNNc can be used to analyse the spatio-temporal segmentation of the activated brain regions. Each colour represents a distinct segmented area of the brain based on the spatio-temporal similarity.

Fig.9 and Fig. 10 represent the spatio-temporal connections between 5062 spiking neurons after the NeuCube training process by affirmative sentence stimulus versus negative sentence stimulus respectively. The SNNc was constructed based on the star/plus voxel coordinates and plotted in 2D.

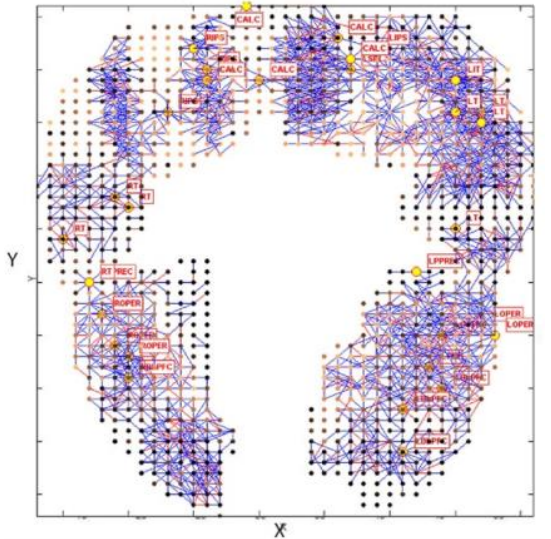

Fig. 9. Neuron connections generated by affirmative sentences

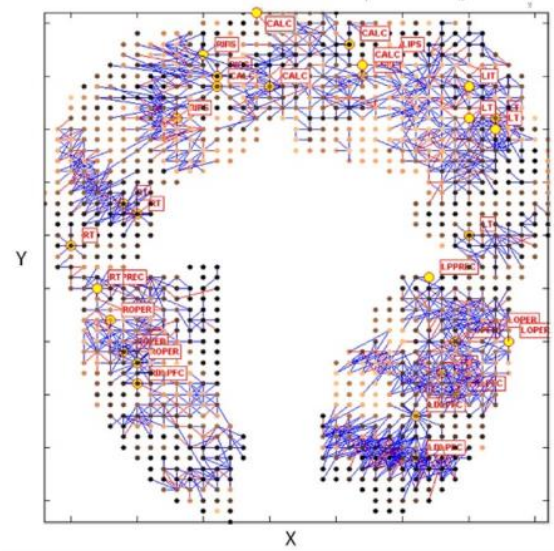

Fig. 10. Neuron connections generated by negative sentences.

\section{B. EEG data classification using deSNN classifier of the NeuCube}

In this study, the classification results were evaluated using both repeated random rub-sampling cross validation (RRSV) and leave-one-out cross validation (LOOCV). In this experiment, the RRSV method has been applied with $50 \%$ training data and $50 \%$ test data. In order to optimize the classification accuracy, we have altered the value of three selected parameters (AER threshold, Connection distance and STDP rate). After a total number of 1000 model generated, the best accuracy was reported. The parameters that generated the best classification accuracy are reported in Table 2 .

\begin{tabular}{|c|c|c|c|c|c|}
\hline \multicolumn{1}{|c}{ Table II. Parameter setting of the NeuCube } \\
\hline & $\begin{array}{c}\text { AER } \\
\text { threshold }\end{array}$ & $\begin{array}{c}\text { Connection } \\
\text { distance }\end{array}$ & $\begin{array}{c}\text { STDP } \\
\text { rate }\end{array}$ & $\begin{array}{c}\text { Firing } \\
\text { threshold }\end{array}$ & $\begin{array}{c}\text { deSNN } \\
\text { mod }\end{array}$ \\
\hline Session I & 3.327 & 0.128 & 0.010 & 0.5 & 0.4 \\
\hline Session II & 2.852 & 0.125 & 0.013 & 0.5 & 0.4 \\
\hline Session III & 2.0929 & 0.108 & 0.014 & 0.5 & 0.4 \\
\hline
\end{tabular}

Table 3 summarised the fMRI data classification accuracy of affirmative sentences class versus negative sentences class obtained using RRSV and LOOCV evaluation methods of the NeuCube-based model. Table 4 reports the classification accuracy results attained via traditional machine learning methods simulated in NeuCome, such as Support Vector 
Machine (SVM), Multiple Linear Regression (MLR), MultiLayer Perceptron (MLP), Evolving Classification Function (ECF), and Evolving Clustering Methods (ECMC). Table 5 demonstrates the recently published classification results of the affirmative sentences versus negative sentences on the same fMRI data [3]. These results achieved via SVM.

Table III. Classification accuracy of affirmative/negated classes using RRSV and LOOCV of the NeuCube .The sample file contains 40 examples (20 examples per class). Confusion table is reported on the right column to show misclassified samples after the testing (in RRSV $50 \%$ of the data used for testing: 10 out of 20 )

\begin{tabular}{|c|c|c|c|c|c|c|c|}
\hline \multirow{3}{*}{$\begin{array}{c}\text { NeuCube } \\
\text { validation } \\
\text { method }\end{array}$} & \multicolumn{7}{|c|}{ The classification accuracy resulted from NeuCube-based model } \\
\hline & \multirow[t]{2}{*}{ Brain region (number of voxels) } & \multicolumn{3}{|c|}{ Accuracy } & \multirow{2}{*}{\multicolumn{3}{|c|}{ Confusion table }} \\
\hline & & $\mathbf{C 1}$ & $\mathrm{C2}$ & Total & & & \\
\hline \multirow{11}{*}{ RRSV } & \multirow{3}{*}{$\begin{array}{l}\text { Session I: 'LSGA'(1), 'LT' (3), LOPER '(3) } \\
\text { 'LIPL'(1), LDLPFC' (6) 'RT'(2), 'CALC'(1)'RIT'(1) } \\
\text { 'RDLPFC'(1), 'RSGA' (1), }\end{array}$} & \multirow{3}{*}{$80 \%$} & \multirow{3}{*}{$100 \%$} & \multirow{3}{*}{$90 \%$} & & C1 & C2 \\
\hline & & & & & C1 & 8 & 2 \\
\hline & & & & & $\mathbf{C 2}$ & 0 & 10 \\
\hline & & & & & & & \\
\hline & \multirow{3}{*}{ Session II: RDLPFC (20) } & \multirow{3}{*}{$90 \%$} & \multirow{3}{*}{$80 \%$} & \multirow{3}{*}{$85 \%$} & & $\mathrm{C} 1$ & $\mathrm{C} 2$ \\
\hline & & & & & C1 & 9 & 1 \\
\hline & & & & & $\mathrm{C2}$ & 2 & 8 \\
\hline & \multirow{4}{*}{ Session III: LDLPFC (20) } & \multirow{4}{*}{$90 \%$} & & & & & \\
\hline & & & \multirow{3}{*}{$80 \%$} & \multirow{3}{*}{$85 \%$} & & C1 & $\mathrm{C2}$ \\
\hline & & & & & C1 & 9 & 1 \\
\hline & & & & & $\mathrm{C2}$ & 2 & 8 \\
\hline & & & & & & & \\
\hline \multirow{3}{*}{ LOOCV } & \multirow{3}{*}{$\begin{array}{l}\text { 'LSGA'(1), 'LT' (3), LOPER '(3) } \\
\text { 'LIPL'(1), LDLPFC' (6) 'RT'(2), 'CALC'(1)'RIT'(1) } \\
\text { 'RDLPFC'(1), 'RSGA' (1), }\end{array}$} & \multirow{3}{*}{$60 \%$} & \multirow{3}{*}{$50 \%$} & \multirow{3}{*}{$55 \%$} & & C1 & $\mathbf{C 2}$ \\
\hline & & & & & C1 & 12 & 8 \\
\hline & & & & & $\mathrm{C2}$ & 10 & 10 \\
\hline
\end{tabular}

Table IV. Classification accuracy for affirmative/negated sentences obtained using traditional machine learning methods (obtained via NeuCom).

\begin{tabular}{|c|c|c|c|c|c|c|c|c|}
\hline \multirow[t]{2}{*}{ Method } & \multirow[t]{2}{*}{ Parameter setting } & \multicolumn{3}{|c|}{ Accuracy } & \multicolumn{3}{|c|}{ Confusion table } & $\begin{array}{c}\text { Class } \\
\text { Performance }\end{array}$ \\
\hline & & C1 & $\mathrm{C2}$ & total & & & & \multirow{4}{*}{0.05} \\
\hline \multirow{3}{*}{ SVM } & \multirow{3}{*}{$\begin{array}{l}\text { Number of features used: All Features } \\
\text { SVM Kernal: Polynomial Degree, } \\
\text { Gamma, N/A : } 1\end{array}$} & \multirow{3}{*}{$70 \%$} & \multirow{3}{*}{$75 \%$} & \multirow{3}{*}{72.50} & & C1 & $\mathrm{C2}$ & \\
\hline & & & & & C1 & 14 & 6 & \\
\hline & & & & & $\mathrm{C2}$ & 5 & 15 & \\
\hline & & & & & & & & \\
\hline \multirow{3}{*}{ MLP } & \multirow{3}{*}{$\begin{array}{l}\text { Number of features used: All Features } \\
\text { Number of Hidden Units: } 5 \\
\text { Number of Training Cycles: } 600 \\
\text { Output Value Precision:0.0001 } \\
\text { Output Function Precisio:0.0001 } \\
\text { Output Activation Function: linear } \\
\text { Optimisation : scg }\end{array}$} & \multirow[b]{3}{*}{$70 \%$} & \multirow[b]{3}{*}{$75 \%$} & \multirow[b]{3}{*}{$72 \%$} & & C1 & $\mathrm{C2}$ & \multirow[b]{3}{*}{0.05} \\
\hline & & & & & C1 & 14 & 6 & \\
\hline & & & & & $\mathrm{C2}$ & 5 & 15 & \\
\hline & & & & & & & & \\
\hline \multirow{3}{*}{ MLR } & \multirow{3}{*}{ Number of features used: 20} & \multirow{3}{*}{$65 \%$} & \multirow{3}{*}{$60 \%$} & \multirow{3}{*}{$62 \%$} & & C1 & $\mathrm{C2}$ & \multirow{3}{*}{0.26} \\
\hline & & & & & C1 & 13 & 7 & \\
\hline & & & & & $\mathrm{C2}$ & 8 & 12 & \\
\hline & & & & & & & & \\
\hline \multirow{3}{*}{ ECF } & \multirow{3}{*}{$\begin{array}{l}\text { Number of features used: } 20 \\
\text { Maximum Influence Field: } 1 \\
\text { Minimum Influence Field: } 0.01 \\
\text { M of N: } 3 \\
\text { Number of Membership Functions: } 2 \\
\text { Number of Epochs: } 4\end{array}$} & \multirow[b]{3}{*}{$55 \%$} & \multirow[b]{3}{*}{$70 \%$} & \multirow[b]{3}{*}{$62 \%$} & & $\mathrm{C} 1$ & $\mathrm{C} 2$ & \multirow[b]{3}{*}{0.17} \\
\hline & & & & & C1 & 11 & 9 & \\
\hline & & & & & $\mathrm{C2}$ & 6 & 14 & \\
\hline \multirow{3}{*}{ ECMC } & \multirow{3}{*}{$\begin{array}{l}\text { Number of features used: } 30 \\
\text { Maximum Influence Field }: 2 \\
\text { Minimum Influence Field }: 0.01 \\
\text { M of N : } 3\end{array}$} & \multirow{3}{*}{$65 \%$} & \multirow{3}{*}{$70 \%$} & \multirow{3}{*}{$70 \%$} & & C1 & $\mathrm{C2}$ & \multirow{3}{*}{0.05} \\
\hline & & & & & $\mathbf{C 1}$ & 13 & 7 & \\
\hline & & & & & $\mathrm{C2}$ & 6 & 14 & \\
\hline
\end{tabular}

Tabless V. The classification accuracy results published in previous study on the same fMRI data set using the SVM [3].

\begin{tabular}{|c|c|c|c|c|c|c|c|}
\hline & Brain region & & Accuracy & & & fusion & \\
\hline & & C1 & $\mathrm{C2}$ & total & & & \\
\hline \multirow{7}{*}{$\begin{array}{l}\text { SVM classification } \\
\text { accuracy results } \\
\text { published in [3] }\end{array}$} & \multirow{3}{*}{ Session I: RDLPFC } & \multirow{3}{*}{$63.33 \%$} & \multirow{3}{*}{$67.81 \%$} & \multirow{3}{*}{$65.57 \%$} & & C1 & $\mathrm{C2}$ \\
\hline & & & & & C1 & 63.33 & 36.63 \\
\hline & & & & & $\mathrm{C2}$ & 32.19 & 67.81 \\
\hline & & & & & & & \\
\hline & \multirow[t]{3}{*}{ Session II: RDLPFC } & \multirow{3}{*}{$64.08 \%$} & \multirow{3}{*}{$68.44 \%$} & \multirow{3}{*}{$66.26 \%$} & & C1 & $\mathrm{C2}$ \\
\hline & & & & & C1 & 64.08 & 35.92 \\
\hline & & & & & $\mathbf{C 2}$ & 31.56 & 68.44 \\
\hline
\end{tabular}




\section{CONCLUSION}

In this study, we have used fMRI data using NeuCube evolving SNN model to analyse the evoked areas of the brain while a human subject was reading different sentences. The experimental results proved the following phenomena:

The brain activity patterns of the subject performed differently in affirmative versus negative sentence. With the proposed NeuCube-based model, we obtained higher classification accuracy in comparing with previous study that used the SVM classifier on the same fMRI data proposed in [3] and also traditional machine learning methods.

The overall classification accuracy achieved during the experimental session $I$ was the highest. $100 \%$ of the voxel activity patterns corresponding to the negative sentence stimulus were classified correctly; while, the accuracy of the affirmative sentence stimulus was $80 \%$. We can conclude that the brain activity patterns of negated sentences are more distinguishable than affirmative sentence.

Through the visualisation of the SNNc, we can observe that the NeuCube is able to evolve in size to adapt different STBD. Also, the visualisation proved that the left hemisphere (LDLPFC and LT) was more activated than right hemisphere (RDLPFC and RT) while reading a negative sentence stimuli.

\section{FUTURE WORKS}

Further improvement and development of the proposed NeuCube-based model, are believed to significantly contribute to the advancement in machine learning for the classification, segmentation and understanding of fMRI spatio-temporal brain data. For this reason, appropriate methods for fMRI data segmentation in terms of both spatial and temporal similarities will be defined.

\section{ACKNOWLEDGMENT}

The research is supported by the Knowledge Engineering and Discovery Research Institute of the Auckland University of Technology (www.kedri.aut.ac.nz). The authors would like to acknowledge all the researchers that have contributed to the realization of this study.

\section{REFERENCES}

[1] N. Kasabov, "NeuCube: A spiking neural network architecture for mapping, learning and understanding of spatio-temporal brain data," Neural Networks, vol. 52, pp. 62-76, 2014.

[2] N. Kasabov, K. Dhoble, N. Nuntalid and G. Indiveri, "Dynamic evolving spiking neural networks for on-line spatio-and spectrotemporal pattern recognition," Neural Networks, vol. 41, pp. 188201, 2013

[3] M. Behroozi and M. R. Daliri, "RDLPFC area of the brain encodes sentence polarity: a study using fMRI," Brain imaging and behavior, pp. 1-12, 2014

[4] M. A. Lindquist, "The statistical analysis of fMRI data," Statistical Science, vol. 23, pp. 439-464, 2008.

[5] S. Ogawa, . D. W. Tank, . R. Menon, . J. M. Ellermann, S. G. Kim, $\mathrm{H}$. Merkle and K. gurbil, "Intrinsic signal changes accompanying sensory stimulation: functional brain mapping with magnetic resonance imaging," Proceedings of the National Academy of Sciences, vol. 89, no. 13, pp. 5951-5955, 1992

[6] M. Just, "StarPlus fMRI data," [Online]. Available: http://www.cs.cmu.edu/afs/cs.cmu.edu/project/theo-81/www/. [Accessed 1307 2014]

[7] N. Kasabov, Evolving connectionist systems, Springer, 2007

[8] L. Benuskova and N. Kasabov, Computational neurogenetic modeling, New York : Springer, 2007.

[9] W. Gerstner, "What is different with spiking neurons?," in Plausible neural networks for biological modelling., Netherlands, Springer, 2001, pp. 34-48.

[10] H. L. Alan and A. F. Huxley, "A quantitative description of membrane current and its application to conduction and excitation in nerve," The Journal of physiology 1, vol. 117, pp. 500-544, 1952

[11] W. Maass, N. Thomas and M. Henry, "Real-time computing without stable states: a new framework for neural computation based on perturbations," Neural Computation, vol. 14, no. 11, p. 2531-2560, 2002

[12] N. Kasabov and E. Capecci, "Spiking neural network methodology for modelling, classification and understanding of EEG spatiotemporal data measuring cognitive processes," Information Sciences, 2014

[13] N. Kasabov, "NeuCube EvoSpike Architecture for Spatiotemporal," in Artificial Neural Networks in Pattern Recognition, Lecture Notes in Computer Science, 2012.

[14] N. Kasabov, . V. Feigin, Z.-G. Hou, Y. Chen, L. Liang, R. Krishnamurthi, M. Othman and P. Parmar, "Evolving spiking neural networks for personalised modelling, classification and prediction of spatio-temporal patterns with a case study on stroke," Neurocomputing, vol. 134, pp. 269-279, 2014.

[15] K. Dhoble, N. Nuntalid, G. Indiveri and N. Kasabov, "Online spatiotemporal pattern recognition with evolving spiking neural networks utilising address event representation, rank order, and temporal spike learning," in IEEE World Congress on Computational Intelligence, Brisbane, Australia, 2012.

[16] S. Thorpe and G. Jacquse, "Rank order coding," in Computational Neuroscience, 1998.

[17] S. Song, M. D. Kenneth and A. F. Larry, "Competitive Hebbian learning through spike-timing-dependent synaptic plasticity," Nature neuroscience, vol. 3, pp. 919-926, 2000.

[18] E. M. Izhikevich, "Polychronization: Computation with Spikes," Neural Computation, vol. 18, pp. 245-282, 2006.

[19] F. Pereira, "E-print Network," 1302 2002. [Online]. Available: http://www.osti.gov/eprints/topicpages/documents/record/181/3791 737.html. [Accessed 201407 13].

[20] D. I. Hoult and R. Richards, "The signal-to-noise ratio of the nuclear magnetic resonance experiment," Journal of Magnetic Resonance, vol. 24 , no. 1 , pp. $71-85,1976$

[21] "Knowledge Engineering and Discovery Research Institute," [Online]. Available: http://www.kedri.aut.ac.nz/. [Accessed 1307 2014].

[22] J. L. Lancaster, M. G. Woldorff, L. M. Parsons, . M. Liotti, C. S. Freitas, . L. Rainey, . P. . V. Kochunov, D. Nickerson, S. . A. Mikiten and . P. T. Fox, "Automated Talairach Atlas Labels For Functional Brain," Human brain mapping, vol. 10, no. 3, pp. 120--131, 2000.

[23] E. Tu, N. Kasabov, M. Othman, Y. Li, S. Worner, J. Yang and Z. Jia, "NeuCube(ST) for Spatio-Temporal Data Predictive Modelling with a Case Study on Ecological Data," in Neural Networks (IJCNN), Beijing, China, 2014. 